\title{
DNA repair gene XRCC3 T241M polymorphism and susceptibility to hepatocellular carcinoma in a Chinese population: a meta-analysis
}

\author{
R.B. Ji, Y.S. Qian, A.R. Hu and Y.R. Hu \\ Liver Disease Research Center, Ningbo No. 2 Hospital, Ningbo, Zheijiang, China \\ Corresponding authors: A.R. Hu / Y.R. Hu \\ E-mail: huairong1970@126.com
}

Genet. Mol. Res. 14 (4): 15988-15996 (2015)

Received July 25, 2015

Accepted September 30, 2015

Published December 7, 2015

DOI http://dx.doi.org/10.4238/2015.December.7.11

ABSTRACT. Numerous studies have evaluated the association between the X-ray repair complementing defective repair in Chinese hamster cells 3 (XRCC3) T241M polymorphism and hepatocellular carcinoma (HCC) risk. However, the results of such investigations have proved inconsistent. Therefore, we performed a meta-analysis of the association between this polymorphism and HCC risk in the Chinese population. Published literature from PubMed and China National Knowledge Infrastructure databases was retrieved, and a total of 5 case-control studies consisting of 2967 patients and 3874 controls were included in this meta-analysis, which revealed a significant association between the XRCC3 T241M polymorphism and HCC risk (TT vs MM: OR $=6.54,95 \% \mathrm{Cl}=2.14-19.99 ;$ TT vs $\mathrm{MT}: \mathrm{OR}=4.72,95 \% \mathrm{Cl}=2.26-$ 9.86; dominant model: $\mathrm{OR}=0.38,95 \% \mathrm{Cl}=0.26-0.57$; recessive model: $\mathrm{OR}$ $=1.27,95 \% \mathrm{Cl}=0.99-1.62$ ). In a subgroup analysis by sample size (number of subjects > 1000), similar results were obtained. Thus, XRCC3 T241M polymorphism may constitute a risk factor for $\mathrm{HCC}$ in the Chinese population.

Key words: XRCC3; T241M polymorphism; Hepatocellular carcinoma; Meta-analysis 


\section{INTRODUCTION}

Hepatocellular carcinoma ( $\mathrm{HCC}$ ) is the 5 th most common cancer and the $3 \mathrm{rd}$ most frequent cause of cancer-related mortality worldwide, and is particularly prevalent in China (Jemal et al., 2011). Approximately 695,900 people die each year in China from primary carcinoma of the liver, making up almost $45 \%$ of global mortality (Yuen et al., 2009). The development of HCC is linked to an interaction between environmental, dietary, and lifestyle factors. However, despite much investigation, its causes are not yet fully understood. Alcoholism, hepatitis B and C, liver cirrhosis, hemochromatosis, Wilson's disease, and type 2 diabetes are important HCC risk factors (Parkin et al., 2001). In addition, recent studies have investigated the genes underlying the development and progression of $\mathrm{HCC}$, and have proposed that genetic factors may contribute to carcinogenesis (Karabork et al., 2010; Akkiz et al., 2014).

It is now accepted that DNA damage is an important mechanism in the pathogenesis of many cancers including HCC (Tebbs et al., 1995). If damaged DNA is not repaired, mutations and the subsequent development of HCC can occur. DNA repair mechanisms involve nucleotide excision repair, base excision repair, and double-strand break repair (DSBR) pathways (Wood et al., 2001). The DNA repair enzyme X-ray repair complementing defective repair in Chinese hamster cells 3 (XRCC3), a member of the DSBR pathway, plays a direct role in homologous recombination, important for chromosomal integrity and the repair of damaged DNA (Shin et al., 2008; Mao et al., 2014).

The XRCC3 gene is located on chromosome 14q32.33. The most commonly investigated polymorphism of XRCC3, named T241M (rs861539), consists of a C/T transition resulting in an amino acid substitution from Thr to Met at codon 241. Three genotypes have been identified for this polymorphism, namely, wild-type (CC), heterozygote (CT), and homozygote (TT). XRCC3 sequence variations may affect the function of the encoded protein and consequently alter its DNA repair capacity (Matullo et al., 2001). Moreover, recent meta-analyses have suggested that the T241M polymorphism is associated with an increased risk of lung and cervical cancers (Qiu et al., 2013; Qin et al., 2014).

To date, several studies have investigated the relationship between this polymorphism and $\mathrm{HCC}$ risk, but have reached conflicting conclusions. Meta-analysis can be a useful tool in detecting an association with limited sample size, especially in those evaluating rare polymorphisms (Attia et al., 2003). Using relevant studies involving participants of Chinese ancestry, we performed a meta-analysis to establish whether the XRCC3 T241M polymorphism is associated with HCC risk.

\section{MATERIAL AND METHODS}

\section{Selection of studies}

PubMed and China National Knowledge Infrastructure databases were searched to retrieve papers on studies linking XRCC3 T241M polymorphism and HCC risk that were available online by November 2014 and without language restrictions, by using the following key words: "XRCC3", "T241M", "gene polymorphism", "hepatocellular carcinoma/HCC", and "single nucleotide polymorphism". Studies published by the same authors were checked for overlapping participant groups. In the case of partially overlapping study-subject groups, the most recent literature was used. 


\section{Inclusion and exclusion criteria}

To be included in the meta-analysis, studies had to meet the following criteria: i) a casecontrol design including HCC cases and healthy controls; ii) a focus on the association between the T241M polymorphism and susceptibility to HCC; and iii) the inclusion of sufficient genotype data for extraction. The following articles were excluded from the analysis: i) those that were not case-control studies evaluating the association between the T241M polymorphism and HCC risk; ii) case reports, letters, reviews, meta-analyses, and editorial articles; iii) studies that were based on incomplete raw data and those with no usable data reported; and iv) investigations including duplicate data.

\section{Data extraction}

Information was carefully extracted from all eligible publications by two independent reviewers according to the inclusion criteria listed above, and discrepancies were adjudicated by a third reviewer until consensus was achieved on every item. The following information was extracted from each included publication: first author, year of publication, country of the study, number of cases and controls, genotype frequencies in case and control groups, and evidence of Hardy-Weinberg equilibrium (HWE) in the control group.

\section{Statistical analysis}

HWE was assessed by the chi-square test. The association between the XRCC3 T241M polymorphism and HCC was estimated by calculating pooled odds ratios (ORs) and 95\% confidence intervals (Cls) under a co-dominant (TT vs MM, TT vs MT), dominant (MM + MT vs TT), or recessive model (TT + MT vs MM). We quantified the effect of heterogeneity using the $I^{2}$ test, whose value ranges between 0 and $100 \%$ and represents the proportion of inter-study variability that can be attributed to heterogeneity rather than to chance. A fixed-effects model was used for meta-analysis unless an $l^{2}$ value greater than $50 \%$ indicated heterogeneity across studies, in which case a random-effects model was used. In addition, a Galbraith plot was used to identify and exclude those studies responsible for heterogeneity. Sensitivity analysis was principally performed by sequential omission of individual studies or of those containing data that deviated from HWE (Liu et al., 2014). Subgroup analyses were performed based on sample sizes, and publication bias was assessed by visual inspection of funnel plots and the Begg's rank correlation method $(P<0.05$ was considered statistically significant). Moreover, we performed a cumulative meta-analysis to provide a framework to update the observed genetic effect from all studies, and measured the extent to which this changed as evidence accumulated, with a view to establish a trend in estimated risk effect (Zintzaras and Lau, 2008). All analyses were conducted using Stata 12.0 (StataCorp LP, College Station, TX, USA).

\section{RESULTS}

\section{Study characteristics}

Our search strategy retrieved 21 potentially relevant studies. Based on the inclusion criteria, 5 full-text case-control studies were included in the meta-analysis (Long et al., 2008; Liu, 2010; Han et al., 2012; Zeng et al., 2012; Yao et al., 2014) and 16 studies were excluded. A flow 
chart representing the study selection process is shown in Figure 1. The 5 selected articles included a total of $2967 \mathrm{HCC}$ cases and 3874 healthy controls. The publication year of these studies ranged from 2005 to 2014 and all articles were written in English. Control subjects were mainly drawn from healthy populations. The HWE test was performed using the genotype distributions of the control groups, with all being found to be in HWE except for that of Yao et al. (2014). The main characteristics of the eligible studies are summarized in Table 1.

\section{Overall meta-analysis and further subgroup analysis}

The combined results relating to the XRCC3 T241M polymorphism and $\mathrm{HCC}$ risk are summarized in Figure 2 and Table 2. Meta-analysis identified a significant association between this polymorphism and susceptibility to $\mathrm{HCC}$ in Chinese populations (TT vs MM: OR $=6.54,95 \% \mathrm{Cl}=$ 2.14-19.99; TT vs MT: OR $=4.72,95 \% \mathrm{Cl}=2.26-9.86$; dominant model: $\mathrm{OR}=0.38,95 \% \mathrm{Cl}=0.26$ 0.57 ; recessive model: $\mathrm{OR}=1.27,95 \% \mathrm{Cl}=0.99-1.62$ ). Moreover, this significant association was confirmed by the results of the stratified analysis based on sample size (number of subjects $>1000$; TT vs $\mathrm{MM}: \mathrm{OR}=18.45,95 \% \mathrm{Cl}=4.92-69.17$; TT vs MT: $\mathrm{OR}=6.59,95 \% \mathrm{Cl}=2.44-17.78$; dominant model: $\mathrm{OR}=0.08,95 \% \mathrm{Cl}=0.03-0.27$; recessive model: $\mathrm{OR}=3.93,95 \% \mathrm{Cl}=2.56-6.04)$. No reports were excluded based on the Galbraith plot used to analyze heterogeneity (Figure 3 ). The cumulative meta-analysis revealed a trend of increasing estimated risk effect with stable results, demonstrating that the T241M polymorphism was associated with HCC risk (Figure 4).

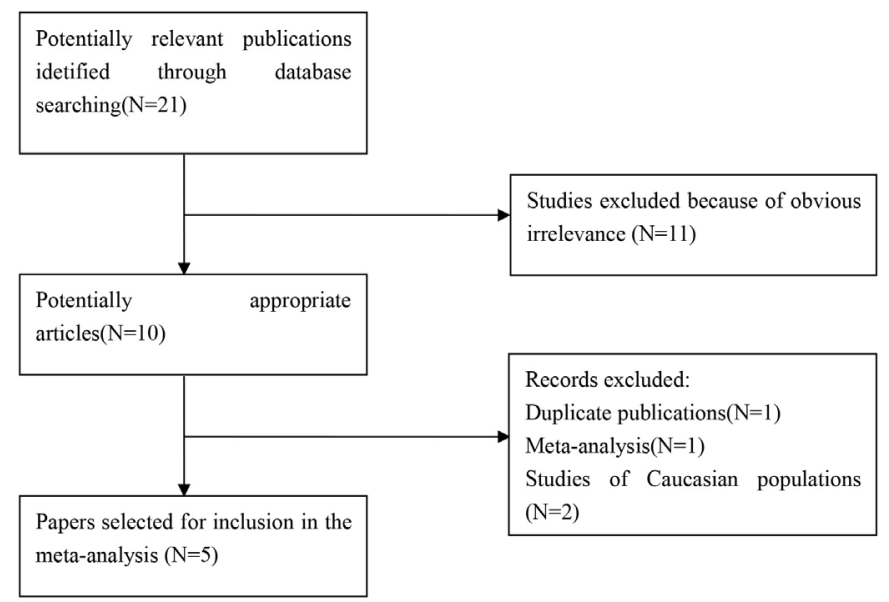

Figure 1. Flow diagram for selection of studies.

Table 1. Characteristics of the studies included in the meta-analysis.

\begin{tabular}{|c|c|c|c|c|c|c|c|c|c|}
\hline \multirow[t]{2}{*}{ Study } & \multirow[t]{2}{*}{ Area } & \multirow[t]{2}{*}{ Cases/Controls } & \multicolumn{3}{|c|}{ Genotypes of cases } & \multicolumn{3}{|c|}{ Genotypes of controls } & \multirow[t]{2}{*}{ HWE test } \\
\hline & & & MM & TM & TT & MM & TM & TT & \\
\hline Long et al. (2008) & Guangxi & $491 / 862$ & 198 & 200 & 93 & 585 & 248 & 29 & 0.67 \\
\hline Liu (2010) & Fuzhou & $344 / 358$ & 319 & 25 & 0 & 337 & 20 & 1 & 0.24 \\
\hline Han et al. (2012) & Luoyang & $149 / 158$ & 75 & 55 & 19 & 87 & 66 & 5 & 0.07 \\
\hline Zeng et al. (2012) & Guangxi & $497 / 500$ & 440 & 50 & 7 & 432 & 65 & 3 & 0.75 \\
\hline Yao et al. (2014) & Shanghai & $1486 / 1996$ & 509 & 634 & 343 & 1430 & 539 & 27 & 0.00 \\
\hline
\end{tabular}

HWE = Hardy-Weinberg equilibrium. 


\section{Sensitivity analysis and publication bias}

Sensitivity analysis was performed by the omission of 1 study containing a dataset that deviated from HWE (Yao et al., 2014). The outcome of the meta-analysis was not altered consequently, demonstrating statistically robust results (Table 2). Moreover, the shape of the Begg's funnel plot suggested that no publication bias was evident (Figure 5).

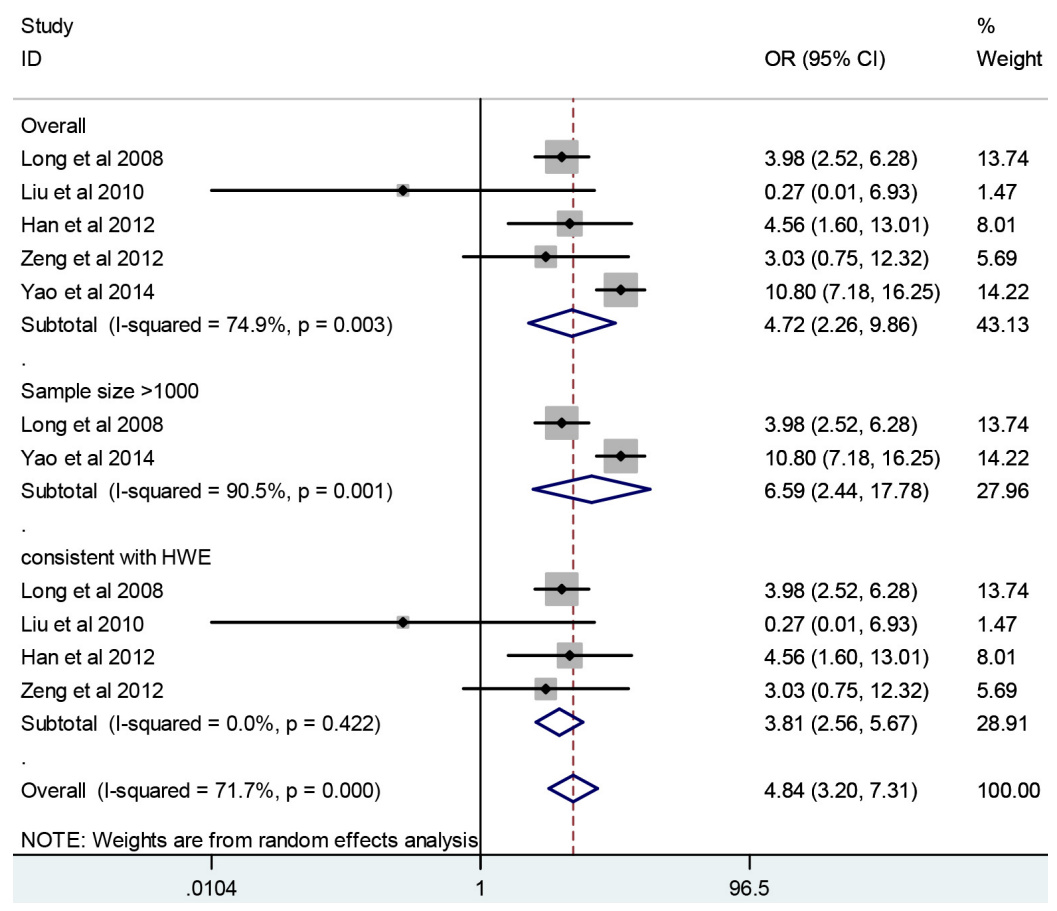

Figure 2. The association between the XRCC3 T241M polymorphism and HCC risk (TT vs MT): total analysis and subgroup analysis by sample sizes $>1000$.

Table 2. Summary of odds ratios and $95 \%$ confidence intervals relating to the XRCC3 T241M polymorphism and hepatocellular carcinoma risk.

\begin{tabular}{|c|c|c|c|c|c|c|c|c|}
\hline \multirow[t]{2}{*}{ Subgroup } & \multirow[t]{2}{*}{ Genetic model } & \multicolumn{2}{|c|}{ Sample size } & \multirow[t]{2}{*}{ Type of model } & \multicolumn{2}{|c|}{ Test of heterogeneity } & \multicolumn{2}{|c|}{ Test of association } \\
\hline & & Cases & Controls & & $P^{2}(\%)$ & $P$ & OR & $95 \% \mathrm{Cl}$ \\
\hline \multirow[t]{4}{*}{ Overall } & TT vs MM & 2967 & 3874 & Random & 90.0 & 0.00 & 6.54 & $2.14-19.99$ \\
\hline & TT vs MT & & & Random & 74.9 & 0.00 & 4.72 & $2.26-9.86$ \\
\hline & Dominant & & & Random & 86.2 & 0.00 & 0.38 & $0.26-0.57$ \\
\hline & Recessive & & & Random & 96.3 & 0.00 & 1.27 & $0.99-1.62$ \\
\hline \multirow[t]{4}{*}{ Sample size $>1000$} & TT vs MM & 1997 & 2858 & Random & 94.8 & 0.00 & 18.45 & $4.92-69.17$ \\
\hline & TT vs MT & & & Random & 90.5 & 0.00 & 6.59 & $2.44-17.78$ \\
\hline & Dominant & & & Random & 93.8 & 0.00 & 0.08 & $0.03-0.27$ \\
\hline & Recessive & & & Random & 90.0 & 0.00 & 3.93 & $2.56-6.04$ \\
\hline \multirow[t]{4}{*}{ Consistent with HWE } & TT vs MM & 1481 & 1878 & Random & 64.7 & 0.04 & 1.25 & $1.62-11.14$ \\
\hline & TT vs MT & & & Fixed & 0.0 & 0.42 & 3.81 & $2.56-5.67$ \\
\hline & Dominant & & & Random & 76.8 & 0.58 & 0.17 & $0.05-0.58$ \\
\hline & Recessive & & & Random & 93.0 & 0.00 & 1.42 & $0.69-2.94$ \\
\hline
\end{tabular}

$\mathrm{OR}$ = odds ratio; $\mathrm{Cl}$ = confidence interval; $\mathrm{HWE}=$ Hardy-Weinberg equilibrium. 


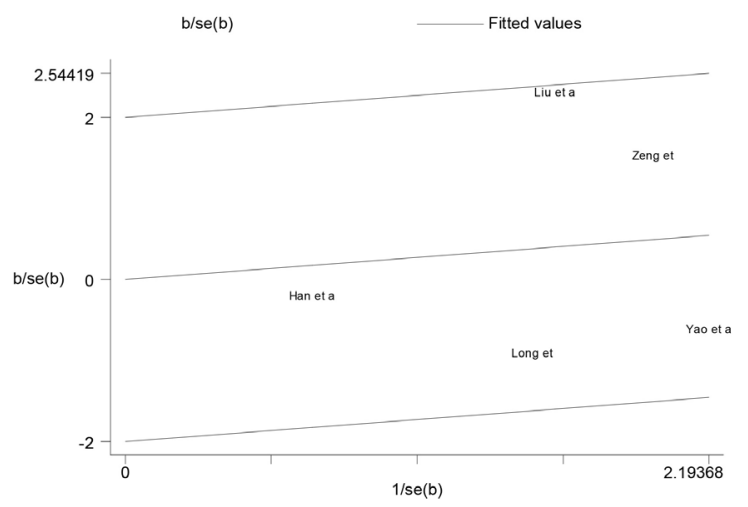

Figure 3. Galbraith plot of studies of the XRCC3 T241M polymorphism and HCC (TT vs MT).

Study
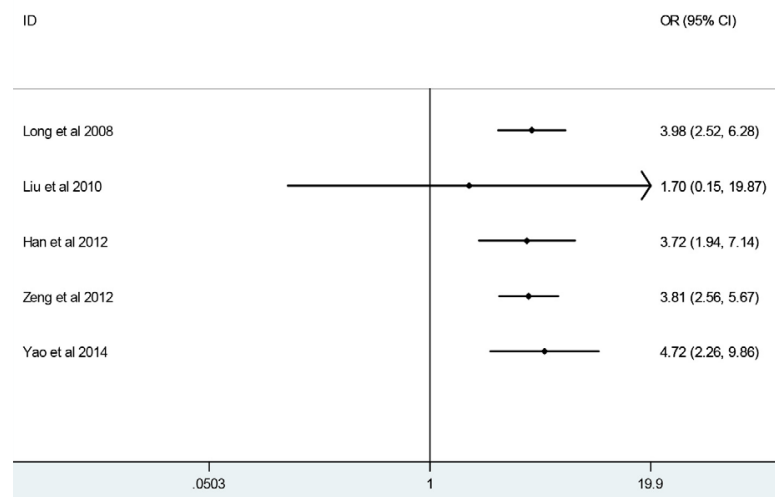

Figure 4. Cumulative meta-analysis for XRCC3 T241M polymorphism and HCC (TT vs MT).

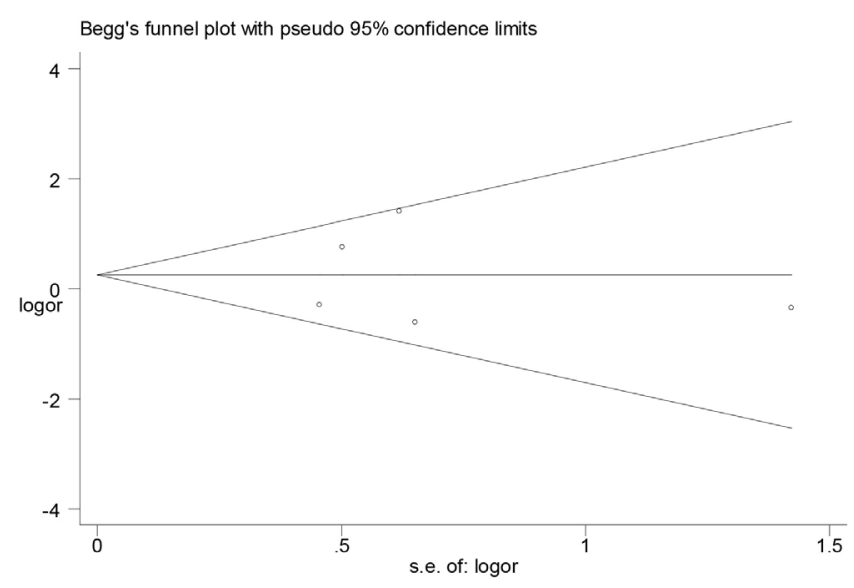

Figure 5. Publication bias for the association between the XRCC3 T241M polymorphism and HCC risk (TT vs MT). 


\section{DISCUSSION}

From a global perspective, China is estimated to have had the highest incidence of HCC in recent years (Parkin et al., 2005). However, the exact mechanism behind HCC pathogenesis remains poorly understood. DNA repair is essential in protecting the genome from environmental hazards. XRCC3 is an RAD51-related enzyme that functions through complex interactions with other proteins to repair double-strand breaks and to maintain genome integrity over multiple phases of homologous recombination (Brenneman et al., 2000). It is well known that single nucleotide polymorphisms are the most common sources of human genetic variation and may contribute to an individual's susceptibility to cancer. Recently, many studies have focused on the association between the XRCC3 T241M polymorphism and HCC, but have generated inconsistent results. The most likely reason for such discrepancies is that the investigations concerned were single case-control studies with small sample sizes. In the present study, we aimed to investigate the relationship between this polymorphism and HCC risk in the Chinese population by meta-analysis.

To the best of our knowledge, this work constitutes the first meta-analysis investigating the association between the XRCC3 T241M polymorphism and HCC risk in the Chinese population. Ultimately, 5 case-control studies were included and assessed, encompassing a total of $2967 \mathrm{HCC}$ patients and 3874 healthy controls, with the study population being confined to participants of Chinese descent with homogeneous genetic backgrounds. The main meta-analysis results showed a significant association between the XRCC3 T241M polymorphism and HCC risk. In addition, data from the cumulative meta-analysis were sufficient to suggest a statistically significant association. As some of the included studies used small sample sizes, this might have skewed the results. However, a subgroup analysis based on sample size revealed the same association, suggesting an absence of small-study bias in our meta-analysis. The deviation of allelic distributions from HWE may contribute to inter-study heterogeneity. A sensitivity test limiting the dataset to include only studies containing distributions consistent with HWE demonstrated that our meta-analysis was accurate and credible. Importantly, there was no evidence of publication bias in this meta-analysis.

The mechanism by which the XRCC3 T241M polymorphism influences HCC risk remains unclear. This polymorphism may alter XRCC3 function, diminishing DNA repair kinetics, and thereby influencing susceptibility to adverse outcomes such as HCC. The potential influence of the T241M variant may depend on gene-gene and gene-environment interactions, and XRCC3 haplotypes (T241M, rs12432907, and rs861537) might act synergistically to increase the risk of developing HCC (Luo et al., 2014). In addition, a previous stratified analysis indicated that the XRCC3 T241M polymorphism is significantly associated with HCC risk among hepatitis B surface antigen-positive individuals and those who consume alcohol (Han et al., 2012). Owing to insufficient available data, further gene-environment interaction studies should be taken into consideration in future analyses.

Moreover, some limitations of this meta-analysis should be taken into account. First, the sample size was still relatively small and may not have provided sufficient power to firmly establish the association between the XRCC3 T241M polymorphism and HCC risk. Therefore, a greater number of studies with larger sample sizes are needed to provide a more accurate, representative statistical analysis (Xiao et al., 2014). Second, owing to limitations regarding literature quality, we were unable to use meta-regression analysis to investigate sources of heterogeneity. Instead, a Galbraith plot was constructed but no studies were excluded, therefore further relevant reports are needed. Third, the effects of gene-gene and gene-environment interactions were not addressed in this meta-analysis. 
In conclusion, our meta-analysis indicates that the XRCC3 T241M polymorphism might be associated with an increased risk of HCC in the Chinese population. However, considering the limitations of the present work, it is necessary to conduct further research with standardized and unbiased methods, larger sample sizes, and well-matched controls.

\section{Conflicts of interest}

The authors declare no conflict of interest.

\section{REFERENCES}

Akkiz H, Kuran S, Akgöllü E, Usküdar O, et al. (2014). The role of interleukin 28B gene polymorphism in Turkish patients with hepatocellular carcinoma. Ann. Hepatol. 13: 788-795.

Attia J, Thakkinstian A and D'Este C (2003). Meta-analyses of molecular association studies: methodologic lessons for genetic epidemiology. J. Clin. Epidemiol. 56: 297-303.

Brenneman MA, Weiss AE, Nickoloff JA and Chen DJ (2000). XRCC3 is required for efficient repair of chromosome breaks by homologous recombination. Mutat. Res. 459: 89-97.

Han X, Xing Q, Li Y, Sun J, et al. (2012). Study on the DNA repair gene XRCC1 and XRCC3 polymorphism in prediction and prognosis of hepatocellular carcinoma risk. Hepatogastroenterology 59: 2285-2289.

Jemal A, Bray F, Center MM, Ferlay J, et al. (2011). Global cancer statistics. CA Cancer J. Clin. 61: 69-90.

Karabork A, Kaygusuz G and Ekinci C (2010). The best immunohistochemical panel for differentiating hepatocellular carcinoma from metastatic adenocarcinoma. Pathol. Res. Pract. 206: 572-577.

Liu W (2010). The relation between genetic polymorphism of DNA repair gene XRCC3, XRCC4 and susceptibility to primary hepatocellular carcinoma. Master's thesis, Fujian Medical University, Fuzhou.

Liu DJ, Liu Y, Ran LM and Li DT (2014). Genetic variants in interleukin genes and susceptibility to IgA nephropathy: A metaanalysis. DNA Cell Biol. 33: 345-354.

Long XD, Ma Y, Qu de Y, Liu YG, et al. (2008). The polymorphism of XRCC3 codon 241 and AFB1-related hepatocellular carcinoma in Guangxi population, China. Ann. Epidemiol. 18: 572-578.

Luo HC, Zhang HB, Xin XJ and Huang WX (2014). Haplotype-based case-control study of DNA repair gene XRCC3 and hepatocellular carcinoma risk in a Chinese population. Tumour Biol. 35: 3415-3419.

Mao CF, Qian WY, Wu JZ, Sun DW, et al. (2014). Association between the XRCC3 Thr241Met polymorphism and breast cancer risk: an updated meta-analysis of 36 case-control studies. Asian Pac. J. Cancer Prev. 15: 6613-6618.

Matullo G, Guarrera S, Carturan S, Peluso M, et al. (2001). DNA repair gene polymorphisms, bulky DNA adducts in white blood cells and bladder cancer in a case-control study. Int. J. Cancer 92: 562-567.

Parkin DM, Bray FI and Devesa SS (2001). Cancer burden in the year 2000. The global picture. Eur. J. Cancer 37: S4-S66.

Parkin DM, Bray F, Ferlay J and Pisani P (2005). Global cancer statistics, 2002. CA Cancer J. Clin. 55: 74-108.

Qin LY, Chen X, Li P, Yang Z, et al. (2014). Association between the XRCC3 Thr241Met polymorphism and cervical cancer risk: a meta-analysis. Asian Pac. J. Cancer Prev. 14: 6703-6707.

Quu M, Xu L, Yang X, Ding X, et al. (2013). XRCC3 Thr241Met is associated with response to platinum-based chemotherapy but not survival in advanced non-small cell lung cancer. PLoS One 8: e77005.

Shin A, Lee KM, Ahn B, Park CG, et al. (2008). Genotype-phenotype relationship between DNA repair gene genetic polymorphisms and DNA repair capacity. Asian Pac. J. Cancer Prev. 9: 501-505.

Tebbs RS, Zhao Y, Tucker JD, Scheerer JB, et al. (1995). Correction of chromosomal instability and sensitivity to diverse mutagens by a cloned cDNA of the XRCC3 DNA repair gene. Proc. Natl. Acad. Sci. U. S. A. 92: 6354-6358.

Wood RD, Mitchell M, Sgouros J and Lindahl T (2001). Human DNA repair genes. Science 291: 1284-1289.

Xiao Y, Deng TR, Su CL and Shang Z (2014). Methylenetetrahydrofolate reductase polymorphisms and susceptibility to acute lymphoblastic leukemia in a Chinese population: a meta-analysis. Oncol. Res. Treat. 37: 576-582.

Yao JG, Huang XY and Long XD (2014). Interaction of DNA repair gene polymorphisms and aflatoxin B1 in the risk of hepatocellular carcinoma. Int. J. Clin. Exp. Pathol. 7: 6231-6244.

Yuen MF, Hou JL, Chutaputti A and Asia Pacific Working Party on Prevention of Hepatocellular Carcinoma (2009). Hepatocellular carcinoma in the Asia pacific region. J. Gastroenterol. Hepatol. 24: 346-353.

Zeng X, Liu S, Yu H, Ji L, et al. (2012). DNA repair capacity, DNA-strand break repair gene polymorphisms, and the incidence of hepatocellular carcinoma in southwestern Guangxi of China. DNA Cell Biol. 31: 1384-1391.

Zintzaras E and Lau J (2008). Synthesis of genetic association studies for pertinent gene-disease associations requires appropriate methodological and statistical approaches. J. Clin. Epidemiol. 61: 634-645. 